\title{
El cacao, de recurso agrario a potencial turístico cultural. Motivaciones de sus productores en la zona centro norte de Manabí, Ecuador
}

\author{
Cocoa from agricultural resource to cultural tourism potential. \\ Producers motivations in the north central area of Manabí, Ecuador
}

\author{
Ángel Guillermo Félix Mendoza* \\ Nelson García Reinoso \\ Ángel Frowen Cedeño Sacón \\ Wilson Paúl Cedeño Guzmán
}

Escuela Superior Politécnica Agropecuaria de Manabí "Manuel Félix López", Ecuador

\section{RESUMEN}

Se presenta la situación productiva del cacao en la provincia de Manabí (Ecuador), así como sus posibilidades de desarrollo regido por una política de revalorización cultural sostenible. Los productores de cacao, por lo general, desarrollan sus actividades en zonas rurales y periferias urbanas, las condiciones de vida muchas veces son precarias; el 90\% de sus unidades productivas, denominadas fincas, con una extensión promedio de cinco hectáreas y su mantenimiento se caracteriza por ser de responsabilidad familiar. El objetivo de la presente investigación es identificar los diferentes grupos de productores, a partir de sus motivaciones, para el cultivo y producción del cacao en la zona centro norte de Manabí, Ecuador. El trabajo se dividió en dos etapas: (1) exploratoria, mediante la utilización de datos provenientes de organismos oficiales, los mismos que fueron procesados con el software ARC GIS; (2) trabajo de campo utilizando un cuestionario con escala de Likert. La muestra está conformada por 196 productores de cacao. El diseño de investigación es flexible, de enfoque cuantitativo y cualitativo; de carácter exploratorio. Existen dos grandes conjuntos de productores. El primer conjunto de productores relacionado con la simple comercialización del cacao y el segundo conjunto de productores que muestra una significante apropiación del producto como reflejo de la cultura local. Se vislumbra un contexto positivo para las iniciativas en torno al turismo agrario.

Palabras clave: agroturismo, recurso cultural, patrimonio agrario, turismo rural, paisajes, culturales.

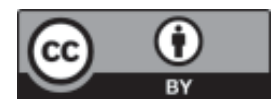




\begin{abstract}
The situation of the production of cocoa in the province of Manabi (Ecuador) is presented, together with its development possibilities with the introduction of a sustainable cultural revaluation policy. Cocoa producers generally carry out their activities in rural areas and urban peripheries where living conditions are often precarious; each productive unit (farm) has an approximately extension of five hectares, and 90\% of their maintenance is carried out by the family. The objective of this research was to identify the different groups of producers, based on their motivations, for the production of cocoa in the north central area of Manabi, Ecuador. The work comprised two stages: (1) exploratory, where data from official bodies was processed on the ARC GIS software; (2) field work, in which a Likert scale questionnaire was used. The sample is made up of 196 cocoa producers. The research design is flexible, with a quantitative and qualitative approach; exploratory in nature. There are two large groups of producers. The first set of producers related to the simple commercialization of cocoa and the second set of producers that shows a significant appropriation of the product as a reflection of the local culture. A positive context is glimpsed for initiatives around agricultural tourism.
\end{abstract}

Keywords: agro-tourism, cultural resource, agrarian patrimony, rural tourism, cultural, landscapes.

\title{
Introducción
}

La situación productiva del cacao en la provincia de Manabí, Ecuador; así como las posibilidades de desarrollo regido por una política de revalorización cultural sostenible merecen la atención de los organismos de gobierno local y de las instituciones de protección e investigación. En los sistemas agrarios de Ecuador, donde los productores de cacao, por lo general, desarrollan sus actividades en zonas rurales y periferias urbanas, las condiciones de vida muchas veces son precarias; se encuentran índices alarmantes como la tasa de pobreza que llega a 52\% en 2014. El 90\% de sus unidades productivas, denominadas fincas, poseen una extensión promedio de cinco hectáreas y su mantenimiento se caracteriza por ser de responsabilidad familiar. Como resultado tienen baja productividad, incidiendo en aspectos sociales y económicos que conllevan a la búsqueda de otras vías de explotación agraria, no siempre sostenibles; por el contrario, muchas veces provocan excesiva presión sobre los recursos disponibles en el territorio, entre estos la tierra, la biodiversidad y los bosques (Lehman y Springer-Heinze, 2014).

En este contexto, se advierte que la mayor cantidad de productores carecen de facilidades para mejorar la producción de sus cultivos, como sucede con el cacao. No hay renovación en sus plantaciones y se ven limitados para obtener variedad de cacao, lo que dificulta tener más elementos para competir en el mercado mundial. En el aspecto sociocultural, también se limita la participación de la mujer en la cadena de valor, debido a las costumbres transmitidas de generación en generación, en la que primordialmente es el varón quien se encarga de la producción y comercialización de los productos (Barrezueta-Unda y Paz, 2017). Sin embargo, en lo comercial es impresionante la variedad de cacao que se cultiva en Ecuador, en contraste con la mayoría de países productores de este rubro que cultivan el denominado cacao común. En los últimos años, Ecuador se ha convertido en el principal país productor mundial de Cacao Nacional Fino y de Aroma (CNFA) o de Cacao 
Arriba, con su calidad genética reconocida internacionalmente. También, paralelamente a estos cultivos, crecen otras variedades híbridas, no tan valoradas en el ámbito chocolatero (Vassallo, 2017).

Con respecto a la exportación del cacao en grano del país, la Asociación Nacional de Exportadores de Cacao (ANECACAO, 2016), registra entre sus cifras anuales de exportaciones del rubro cacao, volúmenes generales de 260000 toneladas métricas de granos de cacao y productos varios derivados de este. Las variedades de cacao exportadas, según nomenclatura utilizada en la Norma Técnica Ecuatoriana INEN 176 es como sigue: 47\% de cacao nacional fino y de aroma A.S.E. (Arriba Superior Época); 30\% de CCN51 (Colección Castro Naranjal árbol 51); 18\% de cacao fino y de aroma A.S.S. (Arriba Superior Selecto); 5\% de cacao fino y de aroma A.S.S.S. (Arriba Superior Summer Selecto); y solo 0.8\% de productos con valor agregado como chocolates, barras, tabletas, coberturas y bombones (ANECACAO, 2016). Cifras que muestran una gran desproporción entre la exportación de granos y productos terminados; esto genera gran debate dentro del territorio debido a que la cadena productiva es muy corta. Los procesos con mayores problemas son los de fermentación y comercialización. Es preciso recalcar que la mayoría de organizaciones agroindustriales relacionadas al cacao se enfrentan a problemas fitosanitarios, principalmente los correspondientes a variedades de tipo nacional, debido a malas prácticas agrícolas tradicionales, sobre todo en la etapa de postcosecha donde los malos manejos en la fermentación están provocando una baja de calidad del producto final para exportar (Teneda, 2016).

Cabe destacar que Ecuador es un país tradicionalmente agrícola, en gran parte su desarrollo proviene de la exportación de productos como banano, cacao, café y flores (Rodríguez y Fusco, 2017). Es también considerado como el primer productor mundial de cacao fino y de aroma, aportando el 70\% del producto global, seguido de lejos por Indonesia que contribuye con un 10\% (PRO ECUADOR, 2013).

Por otra parte, el Ministerio de Turismo del Ecuador (MINTUR, 2019), en la nueva planificación turística proyectada hasta el 2030 por PLANDETUR, realiza una breve revisión desde la estadística turística nacional, que está limitada a la información proveniente del Ministerio del Interior en los puntos de acceso al país; junto a pequeños levantamientos de información a lo largo del territorio ecuatoriano durante los días festivos nacionales. $\mathrm{Al}$ respecto se identifica que 58.9\% de los extranjeros que visitan el país tienen una motivación cultural, de los cuales $\mathbf{1 6 . 1 \%}$ visitan la provincia de Manabí; mientras que el turista interno tiene a la provincia de Manabí como segunda opción de viaje en las fiestas nacionales. También, como una posible fuente de desarrollo agroturístico para turismo receptivo debido a la representatividad del producto en mercados internacionales, están las pequeñas comunidades pesqueras en la costa y los pueblos montubios de la zona interior de la provincia.

Como es reconocido, el turismo es una actividad económica con alto grado de sensibilidad, y los efectos de la pandemia por COVID-19 lo han ratificado. La crisis no solo sanitaria, sino también socioeconómica, psicológica; así como las restricciones, han afectado el comportamiento y las preferencias del turista. Frente a esta desconfianza, es necesario seguir normas de bioseguridad y mantenimiento de los establecimientos turísticos con iniciativas creativas como sellos de acreditación COVID-19 free, seguros de viaje que cubran a los viajeros frente a eventuales contagios, etc. (Félix et al., 2021). El objetivo es generar confianza en los consumidores e inversionistas para el turismo rural y cultural, ya que justamente, en esta etapa de 'nueva normalidad' se valora más la naturaleza y los espacios abiertos. Sin duda, la declaratoria de paisajes culturales 
alrededor del cacao en la provincia de Manabí y en todo Ecuador, es una oportunidad ideal para el surgimiento de nuevos destinos que, como anclan, usen la producción del cacao y la trasformación de la materia prima.

Ante esta realidad se requiere contar con ventajas competitivas para la valoración internacional del cacao ecuatoriano. Uno de los primeros intentos es la búsqueda de un modelo de denominación de origen que resalte las características propias según las indicaciones geográficas del territorio. Esto, se consigue mediante la protección de un régimen de propiedad industrial que permita la diferenciación de los productos nacionales relacionados al cacao. Específicamente, la variedad que se pretende fortalecer es la del cacao fino y de aroma, también conocido como cacao nacional o Cacao de Arriba.

La comercialización del cacao fino de aroma ecuatoriano ha planteado un debate en los últimos años dentro del mercado local (Quingaísa y Riveros, 2007). Es así que, dentro del estudio para la denominación de origen del Cacao de Arriba, se exploraron características que lo puedan diferenciar entre las distintas zonas productoras del Ecuador. La investigación genética se centra en identificar sus características organolépticas, lo que sin duda provoca el interés internacional sobre el producto desde varios ámbitos, como el científico, histórico, cultural, comercial, entre otros aspectos que transforman a los territorios productores de cacao en verdaderas zonas de interés para visitar y descubrir. La denominación de origen del cacao del Ecuador supone el acceso a nuevos mercados mediante el valor agregado, generado con la promoción de otros valores característicos del entorno geográfico y social para presentarlo como un producto con garantía de consumo. Han transcurrido años en este esfuerzo y el impacto entre los productores hasta la actualidad no ha trascendido, los mercados internacionales aún tienen sus reservas en función de las evidencias relacionadas a las características organolépticas del producto en el territorio, puntos que hay que seguir fortaleciendo mediante la investigación y, con esto, garantizar la calidad del producto (Quintana y Aguilar, 2018).

Con este antecedente, los estudios sociales relacionados al cacao y a la cultura de sus productores ganan preponderancia, desde hace algún tiempo se pretende dar explicaciones a un sinnúmero de relaciones entre esta actividad productiva y el desarrollo local histórico de sus comunidades. Son variados los estudios dentro del territorio ecuatoriano que relacionan al cacao con disciplinas afines a la cultura y el turismo como la gastronomía, turismo, economía, historia, arqueología, antropología, entre otros; los mismos que se convierten en antecedentes para el presente trabajo (Barrera et al, 2019; Castro et al., 2018; Félix y Bayas, 2016; Instituto Nacional de Meteorología e Hidrología [INAMHI], 2012; Miño, 2015; Paguay, 2012; Vela, 2016; Zambrano, 2014).

En la actualidad, muchas investigaciones culturales y turísticas se encuentran en una búsqueda por lograr un mejoramiento de la puesta en valor del cacao y su cadena productiva. Una oportunidad real dentro del país para interactuar con estos dos rubros sociales es el agroturismo, con el que se puede poner en práctica los principios activos y vivenciales de las modalidades alternativas, y mejorar la puesta en valor de los recursos agrarios de las zonas rurales dedicadas a la producción de este rubro (Félix y Doumet, 2016). Además, el conocimiento de los valores culturales de las comunidades y las estrategias de comercialización mejor adaptadas a la realidad pueden coadyuvar al posicionamiento del producto en nuevos mercados (Galletto, 2018).

Las declaratorias de la UNESCO son una gran oportunidad de desarrollo para nuevos destinos turísticos alrededor del mundo. Esta estrategia implementada en los territorios para promover una valoración diferenciada 
de sus recursos está dando resultados muy positivos; por ejemplo, se cuenta con la denominación Paisajes Culturales, obtenida por el eje cafetalero de Colombia. La UNESCO (2017) define así los paisajes culturales:

Bienes culturales, producto de la acción humana y la naturaleza, que ilustran la evolución de la sociedad a lo largo del tiempo, bajo la influencia de restricciones físicas y/o de las posibilidades de su entorno natural y de las fuerzas sociales, económicas y culturales sucesivas, tanto externas como internas.

\section{La construcción de un paisaje cultural}

Como ejemplo de una declaratoria de paisaje cultural se tiene al Paisaje Cultural Cafetero, con varias miradas y enfoques para su respectivo análisis. Desde la parte teórica, se puede asumir como la puesta en práctica de la valoración de las características patrimoniales de un bien o territorio mediante el desarrollo de una planificación interinstitucional, con la finalidad del desarrollo económico y social de una población determinada. La incorporación de un territorio al imaginario turístico colectivo no es una empresa fácil de llevar a cabo, para su consecución se debe pensar en la transformación de sus recursos turísticos en productos de servucción mediante la comodificación de su patrimonio mediante implementaciones que se adapten a los requerimientos de una determinada demanda (Duis, 2007, p. 71). Pero para lograr la valoración y apropiación de este concepto desde un enfoque social, es necesario implementar un proyecto rector que integre los diferentes actores del territorio donde las comunidades locales sean los sujetos activos, y como resultado de esto sus recursos patrimoniales sean conocidos, valorados y promocionados de manera efectiva, todo esto bajo un modelo de gestión turística eficiente (Gómez, 2015).

Desde otra óptica práctica se puede asumir a la declaratoria del Paisaje Cultural Cafetero Patrimonio de la Humanidad, como una estrategia de desarrollo local, donde a partir de lineamientos claros de porte político, económico y social, se fueron fusionando sobre una misma idea las organizaciones públicas y privadas del territorio, con la finalidad de cohesionar en torno al rubro paisajístico cafetero una cultura y un negocio millonario, otorgando cierto grado de valor a los atributos históricos, culturales, geográficos y económicos, los mismos que fueron consolidándose hasta transformar esa idea inicial en un destino turístico cultural con una alta valoración de su patrimonio a nivel internacional (Mayorga, 2015). La institucionalidad cafetera generada alrededor de la declaratoria bien de la humanidad se consolida en un discurso de corte patrimonial; este bien inmaterial es moldeado y se basa en los valores socioculturales. Los ejes socioculturales propuestos para sostener la propuesta en el tiempo son los siguientes:

- Trabajo familiar, generacional e histórico para la producción de un café de excelente calidad, en el marco de un desarrollo sostenible.

- Cultura cafetera para el mundo.

- Capital social estratégico construido alrededor de una institucionalidad.

- Relación entre tradición y tecnología para garantizar la calidad y sostenibilidad del producto. (Ministerio de Cultura de Colombia, 2011) 
De esta manera el patrimonio se presenta como el elemento de consolidación del territorio, esto conlleva a analizar la inseparable relación entre este tipo de declaratorias y las condiciones que se generan inmediatamente para el turismo. El turismo es un fenómeno o actividad socioeconómica que puede adaptarse a muchos eslabones de la cadena productiva, queda claro que no nace automáticamente con una declaratoria, pero la visibilidad internacional que esta genera, unida a una adecuada promoción de los recursos fue la clave del éxito para que muchos productores de café inviertan en el mejoramiento de sus instalaciones y encuentren en el rubro turístico una posibilidad de desarrollo económico (Mayorga, 2015). En este contexto, cabe destacar el trabajo mancomunado, en el territorio, en torno al valor económico del patrimonio y la fusión de objetivos entre diversas instituciones nacionales como los ministerios de Turismo, Industria y Comercio del país colombiano, logrando que los resultados de la actividad turística en el territorio declarado trasciendan fronteras y el sector se destaque como un destino turístico de clase mundial (Mayorga, 2015).

En cuanto al patrimonio cultural del Ecuador, no se han conseguido aún los objetivos que muestra el país vecino de Colombia, pese a existir declaratorias importantes en el territorio, no se desarrollan los procesos adecuados para que estas trasciendan internacionalmente, la generación de valor agregado mediante el turismo no es la adecuada.

En función de lo expuesto, la presente investigación se suma a otras que pretenden promover exitosamente la cultura en territorios ecuatorianos vinculados a cultivos ancestrales de mucha importancia para la historia nacional, como es el caso del cacao. En muchos pueblos de la costa ecuatoriana, específicamente en la provincia de Manabí, Ecuador, se busca promover una declaratoria que contribuya al reconocimiento de la labor ejercida por sus agricultores, los mismos que tienen una historia viva que contar, este es uno de los principales argumentos para generar una postulación frente a la UNESCO (Félix et al., 2017). La consecución de una declaratoria, acorde con el paisaje cultural del cacao, es una oportunidad importante para potenciar el desarrollo turístico de la costa interna de Manabí, donde existen muchos recursos por desarrollar, y acuñados a esta declaratoria, pueden tener la oportunidad de sobresalir conjuntamente con sus comunidades aledañas. Es una gran oportunidad para fortalecer el desarrollo socioeconómico de este sector agropecuario, teniendo como referente una fórmula que lleve de la mano a los productos agrarios, la cultura alrededor de esta producción y la actividad turística como un eje de desarrollo transversal. La orientación es hacia la exploración de antecedentes históricos y la creación de una línea de tiempo clara sobre la importancia de este rubro para las comunidades locales. Con la información recopilada se puede cumplir con los requisitos del órgano rector, en este caso, UNESCO para postular en la categoría Paisajes Culturales.

Por lo tanto, el objetivo de la presente investigación es identificar los diferentes grupos de productores, a partir de sus motivaciones, para el cultivo y producción del cacao en la zona centro norte de Manabí, Ecuador.

En función del objetivo se plantean seis hipótesis:

H1. Existen diferentes motivaciones para cultivar y producir el cacao en la zona centro norte de Manabí.

H2. Los productores en diferentes componentes muestran diferentes motivaciones para cultivar y producir el cacao en esta zona. 
H3. La segmentación de los productores explica los diferentes niveles de importancia del cacao y su cultura. Específicamente, para cada segmento existen diferencias en la composición del clúster, en relación con: ¿importancia del cacao para padres y abuelos?, ¿cuántas generaciones cultivan el cacao?, ¿qué tan motivado por seguir cultivando?, ¿̇iente al cacao como parte de su identidad como campesinomontubio?, ¿cree usted que nuestro cacao es diferente al de otros lugares?

H4. La segmentación de los productores explica los diferentes niveles de utilización del cacao y su consumo. Existen diferencias significativas en: ¿utilización del cacao?, ¿consume chocolate continuamente?, ¿de qué forma-consume chocolate continuamente?

H5. La segmentación de los productores explica los diferentes niveles de sensaciones de cultivo del cacao. Taxativamente, para cada segmento existen diferencias en relación con las sensaciones que produce su cultivo.

H6. La segmentación de los productores explica los diferentes niveles de su puesta en valor. Particularmente, para cada segmento existen diferencias en relación con: ¿mayor presencia los productos relacionados al cacao?, ¿declaratoria con relación al cacao para Manabí?, ¿cacao como principal producto en los paisajes manabitas?, y ¿estaría dispuesto a formar parte de un circuito y/o producto turístico relacionado al paisaje cultural del cacao?

\section{Metodología}

El diseño de investigación es flexible, de enfoque cuantitativo y cualitativo; de carácter exploratorio. La técnica utilizada es la encuesta y la herramienta para la recolección de la información es el cuestionario con preguntas en escala tipo Likert. La investigación se apoya en un modelo de jerarquización y análisis geográfico sobre plataforma GIS, asociada a programas estadísticos (SPSS v.23) para establecer modelos interpretativos a partir de la gestión de datos.

En la caracterización física del área de estudio, la variante parte de dos elementos, mapa base y capas de contenidos temáticos (MAG, 2019). La georreferenciación se realiza en cinco cantones de la zona centro sur de Manabí, obteniendo 196 puntos que cumplen las condiciones de estudio. Para la realización del mapa temático se emplea la metodología SIGMAT (Jiménez et al., 2010), que se desarrolla en el software ArcGIS versión 10.0 en dos etapas: preparación de cartografía base y de contenidos temáticos. Respecto del entorno social se analiza a las familias que viven en las fincas y la cadena de valor del cacao de la provincia de Manabí (Barrera et al., 2019). Asimismo, se cuenta con fuentes oficiales como el Ministerio de Agricultura y Ganadería y literatura especializada. Y, en relación con la percepción cultural del cacao por parte de los productores, se realiza un análisis estadístico a partir de los resultados en los cuestionarios.

Se considera la población infinita, al no encontrar en Instituciones del sector una estadística fiable de la cantidad de productores de cacao en la zona centro de Manabí. Sin embargo, a partir del Proyecto de Reactivación de Café y Cacao Fino de Aroma (PRCC), se identifican los productores más destacados en 
niveles de producción del territorio. Para determinar el tamaño de la muestra de estudio (ver Tabla 1), se utiliza la metodología propuesta por González y Conde, (2011); Carballo et al., (2016) y García, (2019, 2021); la variabilidad de la población se estima en un 50\% $(p=q=.50)$, valor ampliamente utilizado en investigación social (Carballo, et al., 2016) y se estimó un error muestral del 7\% $(d=.07)$, para un nivel de confianza del 95\% , según la siguiente ecuación:

$$
\begin{gathered}
n=\frac{\mathrm{Z}_{\alpha / 2}^{2} * p * q}{\mathrm{~d}^{2}} \\
n=\frac{1.96^{2} \times 0.5 \times 0.5}{0.07^{2}} \\
n=\frac{0.9604}{0.0049} \\
n=196 \text { encuestas a productores }
\end{gathered}
$$

\section{Tabla 1}

Ficha técnica de la investigación

\begin{tabular}{ll}
\hline Características & Encuestas \\
\hline Población & Productores de cacao mayores de 18 años \\
Ámbito geográfico & Zona centro norte de Manabí \\
Metodología & Encuesta personal mediante cuestionario estructurado \\
Diseño muestral & Muestreo aleatorio sistemático \\
Error de muestreo & $7 \%$ \\
Nivel de confianza & $95 \%(z=1.96)(p=q=.5)$ \\
Tamaño de la muestra & 196 \\
Fecha del trabajo de campo & 1 de diciembre de 2020 al 20 de enero de 2021 \\
\hline
\end{tabular}

La encuesta de 12 preguntas se divide en tres bloques de información: (1) orientado a conocer la valoración acerca del cacao y su cultura, (2) los usos del cacao y (3) la precepción de la puesta en valor del mismo. Se aplicaron determinado número de encuestas a los cantones que dedican más hectáreas al cultivo y son más representativos en la producción de cacao (Barrera et al., 2019). En Bolívar: 39, en Chone: 63, en Flavio Alfaro: 32, en Portoviejo: 30 y en Pichincha: 32. 
En el procedimiento se realizó una segmentación a las comunidades estudiadas, técnica de segmentación frecuentemente utilizada para poder estudiar y analizar necesidades de determinados grupos que comparten ciertos patrones de conductas y estilos de vida; es decir, comprender las motivaciones de estos grupos humanos en cuanto a preferencias y necesidades de los individuos (Andreu et al., 2005). Para obtener la segmentación de los productores de cacao se inició un análisis factorial exploratorio y el cálculo de puntuaciones para clasificar las variables sociales más representativas del productor de cacao en la zona centro norte de Manabí. Además, se aplicó el método ascendente jerárquico (conglomerados, regresión, clúster) para obtener una solución preliminar y seleccionar el número de grupos eliminados. Y, para obtener la clasificación óptima se aplicó el método reasignación K-medias (Aranda et al, 2002; Bonilla et al, 2014; García et al., 2017; Lopes, 2011; Pardo y Del Campo, 2007; Ramirez-Anormaliza et al., 2017).

\section{Discusión}

\section{Caracterización física de la zona de estudio}

Las condiciones climáticas de la zona centro norte de Manabí, lugar donde se ubican los cantones del estudio, tienen temperaturas promedio de 26 a $28{ }^{\circ} \mathrm{C}$ y precipitaciones de 1800 a $2000 \mathrm{~mm}$ anual (INAMHI, 2012). Los cinco cantones más representativos en plantaciones de cacao tienen una superficie total de 93164 ha, como sigue: cantón Chone = 48052 ha; cantón Pichincha = 21378 ha; cantón Flavio Alfaro = 14791 ha; cantón Portoviejo = 2897.7 ha; cantón Bolívar = 6054 ha (Figura 1).

\section{Figura 1}

Caracterización del área objeto de estudio

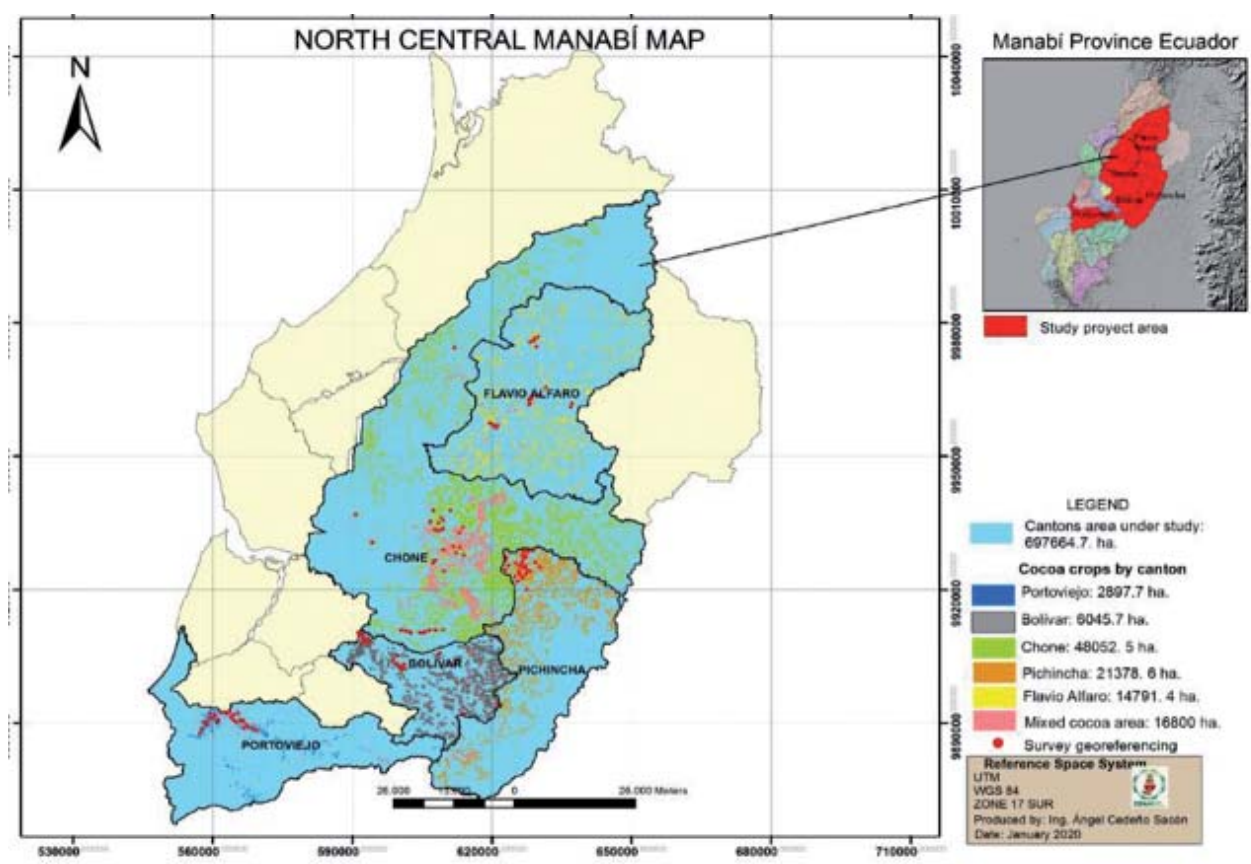




\section{Caracterización social de la zona de estudio}

La conexión entre el territorio de estudio y la producción del cacao se remonta a inicios del siglo XX, donde la demanda externa de los productos de exportación manabitas y la formación de la República del Ecuador crearon una coyuntura favorable que aspiraba a tener en la ciudad de Manta un puerto mayor. Esta categoría permitiría legítimamente importar, exportar y contar con instituciones portuarias, que hasta entonces estaban en Guayaquil, con lo que la provincia se consolidaba en el mapa productivo nacional (Zambrano, 2014). Desde un punto de vista práctico, se desconcentró el proceso comercial y la provincia manabita empezó a tener más autonomía de gestión, lo que contribuyó a la dinamización del territorio y de las comunidades campesinas allí instaladas.

En la actualidad la realidad de las zonas rurales es difícil, fruto de una situación socioeconómica compleja. Se puede observar también un alto índice en la edad y bajo nivel de educación en la población dedicada a la producción cacaotera, que en su mayoría siguen siendo hombres 87.31\% (Barrera et al., 2019). Por otra parte, acerca de la dedicación al cultivo del cacao, se pueden encontrar datos muy alentadores, ya que se observa un promedio de 25 años de experiencia en el cultivo del mismo en las unidades familiares, con una dedicación promedio de tres días y medio a la semana y de casi seis horas diarias en el cultivo, lo que demuestra un alto grado de especialización en la producción del cacao; esto a su vez indica que sigue siendo una de sus actividades económicas de base. Entre otros datos importantes que afianzan esta realidad se tiene que el 99.22\% de los hogares no recibieron remesas externas de ningún tipo y el $80.83 \%$ de las familias no trabajaron en ninguna otra ocupación que no sea la finca (Barrera et al., 2019).

En la provincia de Manabí se destacan tres emprendimientos relacionados con el cacao y el turismo. El primero, la llamada Ruta del cacao de la fundación Eduardo Félix, el segundo es el Circuito del cacao de la Escuela Superior Politécnica Agropecuaria de Manabí Manuel Félix López y el Circuito del cacao del Gobierno Autónomo Descentralizado Provincial de Manabí. En 2018, Félix identifica varias limitaciones para el desarrollo turístico, entre ellas, carencia de empoderamiento en las comunidades locales con respecto a iniciativas; curiosamente, el $84 \%$ de los productores no tiene conocimiento sobre ninguno de estos emprendimientos y se han estancado. Las causas, según el análisis en este estudio, serían los débiles procesos de socialización de estos proyectos y el desconocimiento de factores relacionados a la cultura local.

\section{Resultados}

Existen dos grandes conjuntos de productores. El primer conjunto de productores relacionado con la simple comercialización del cacao y el segundo conjunto de productores que muestra una significante apropiación del producto como reflejo de la cultura local. Las características de cada grupo deben ser tomadas como punto de partida para generar estrategias en los procesos de sensibilización, y así lograr mayor valor agregado del producto a nivel nacional e internacional; también, trabajar para afianzar el sentido de pertenencia de los productores que valoran al cacao como parte de su cultura, mediante proyectos que destaquen la historia en torno a este cultivo, por ejemplo, museos, parques temáticos, circuitos y rutas turísticas, entre otros. 


\section{Percepción cultural del cacao por parte de los productores}

Para definir los segmentos de productores se considera como variable de segmentación las motivaciones en torno al cultivo y la producción del cacao. Se realiza el análisis factorial exploratorio, análisis de los componentes principales y posteriormente un análisis de conglomerados o grupos, método reasignación Kmedias, resultantes de distintas variables relacionadas a la valoración del cacao y su cultura, los usos del cacao y la precepción de la puesta en valor. Mediante el análisis estadístico multivariables se agrupó e identificó las motivaciones para el cultivo y producción del cacao. En la Tabla 2 se muestra que existen diferentes motivaciones para cultivar y producir el cacao en la zona centro norte de Manabí (H1).

\section{Tabla 2}

Análisis factorial de las variables motivaciones para el cultivo y producción del cacao

\begin{tabular}{|c|c|c|c|}
\hline \multirow{2}{*}{ Motivaciones para el cultivo del cacao } & \multicolumn{2}{|c|}{ Componente } & \multirow{2}{*}{ FACTORES } \\
\hline & 1 & 2 & \\
\hline Cultura & .879 & & Factor 1: Cacao-Cultura \\
\hline Identidad & .852 & & \\
\hline Pasión & .844 & & \\
\hline Trabajo & & .858 & Factor 2: Cacao-Trabajo \\
\hline Negocio & & .778 & \\
\hline Sustento diario & & .591 & \\
\hline Autovalores & 2.971 & 1.213 & \\
\hline \% Varianza explicada & 49.522 & 20.213 & \\
\hline$\%$ Varianza acumulada & 49.522 & 69.734 & \\
\hline Alfa de Cronbach & .85 & .79 & \\
\hline KMO (Kaiser-Meyer-Olkin) & & & .785 \\
\hline Prueba de esfericidad de Bartlett & Chi- & rado $=397.176$ & sig. $=.000$ \\
\hline
\end{tabular}

Nota. Método de extracción: Análisis de componentes principales. Método de rotación: Normalización Varimax con Kaisera

El análisis factorial de las variables motivaciones para el cultivo y producción del cacao, evidencian dos factores: Clúster 1: Cacao-Cultura, que representan 43.4\% de los productores de cacao; entre sus motivaciones más representativas para el cultivo y producción del cacao se encuentra: cultura, identidad y pasión. Clúster 2: Cacao-Trabajo, que representan $56.6 \%$ de los productores de cacao; que consideran como sus motivaciones principales para el cultivo y producción del cacao: trabajo, negocio, y sustento diario.

La prueba U de Mann-Whitney se realizó para distinguir las diferencias en las respuestas de los productores. Se obtiene que la media en la motivación-trabajo es la más alta, en comparación con las demás motivaciones seleccionadas por parte de los productores de cacao (Tabla 3). 
Tabla 3

Conglomerado por motivaciones para el cultivo de cacao (Prueba de Mann-Whitney)

\begin{tabular}{|c|c|c|c|c|c|}
\hline \multirow{2}{*}{$\begin{array}{l}\text { Motivaciones para } \\
\text { el cultivo del cacao }\end{array}$} & \multicolumn{2}{|c|}{ Rango promedio } & \multirow{2}{*}{$\begin{array}{c}\text { Media } \\
\text { Muestra }\end{array}$} & \multicolumn{2}{|c|}{ U de Mann-Whitney } \\
\hline & $\begin{array}{c}\text { Factor } 1 \\
\text { Cacao-Cultura }\end{array}$ & $\begin{array}{c}\text { Factor } 2 \\
\text { Cacao-Trabajo }\end{array}$ & & Estadístico & Sig. asintótica \\
\hline Cultura & 146.47 & 61.77 & 7.0663 & 640.000 & .000 \\
\hline Identidad & 148.61 & 60.13 & 6.8010 & 458.000 & .000 \\
\hline Pasión & 150.35 & 58.79 & 5.8265 & 310.000 & .000 \\
\hline Trabajo. & 76.42 & 115.41 & 8.0714 & 2840.500 & .000 \\
\hline Negocio & 73.10 & 117.95 & 7.9337 & 2558.500 & .000 \\
\hline Sustento diario & 70.43 & 120.00 & 7.4031 & 2331.500 & .000 \\
\hline
\end{tabular}

Nota. (1: baja importancia - 10 alta importancia). Kaiser - Meyer - Olkin .785; Chi-cuadrado 397.176; Prueba de esfericidad de Bartlett, $p<.000$

Sobre los diferentes niveles de importancia del cacao y su cultura, se encuentran diferencias significativas con respecto a ¿importancia del cacao para padres y abuelos? $(p<.001)$; ¿cuántas generaciones cultivan el cacao? ( $p<0.001)$; ¿qué tan motivado se encuentra para seguir cultivando? $(p<.006)$; ¿siente al cacao como parte de su identidad como campesino montubio? $(p<.00)$; ¿cree usted que nuestro cacao es diferente al de otros lugares? $(p<.01)$. Se acepta la H3.

Con respecto a las diferencias en los niveles de utilización del cacao y su consumo por parte de los clústeres. Se determina diferencias significativas en la utilización del cacao como comida $(p<.006)$; bebida $(p<.018)$; medicina $(p<.026)$ y negocio $(p<.001)$; así como en ¿consume chocolate continuamente? $(p<.059)$, válido para este estudio exploratorio. No se reflejan diferencias en la utilización del cacao como: arte $(p<.397)$ y cosmético $(p<.909)$; y ¿de qué forma-consume chocolate continuamente? $(p<.174)$. Por lo que la H4 se acepta parcialmente.

En la hipótesis cinco se plantea que la segmentación de los productores explica los diferentes niveles de sensaciones de cultivo del cacao. Taxativamente, para cada segmento existirán diferencias en relación con las sensaciones que produce su cultivo. En la Tabla 4 se muestra que los productores Cacao-Cultura se identifican de manera significativa $(p<.05)$ en los ítems tranquilidad, satisfacción, felicidad y amor. Difieren significativamente de los productores Cacao-Trabajo, que se orientan por otros ítems como la prosperidad y la riqueza. Se acepta la $\mathrm{H} 5$. 
Tabla 4

Sensaciones del productor relacionadas al cacao

\begin{tabular}{|c|c|c|c|c|c|}
\hline \multirow{2}{*}{ Sensaciones } & \multicolumn{2}{|c|}{ Rango promedio } & \multirow{2}{*}{$\begin{array}{c}\text { Media } \\
\text { Muestra }\end{array}$} & \multicolumn{2}{|c|}{ U de Mann-Whitney } \\
\hline & $\begin{array}{c}\text { Factor } 1 \\
\text { Cacao-Cultura }\end{array}$ & $\begin{array}{c}\text { Factor } 2 \\
\text { Cacao-Trabajo }\end{array}$ & & Estadístico & Sig. asintótica \\
\hline Prosperidad & 57.25 & 130.09 & 6.7959 & 1211.500 & .000 \\
\hline Riqueza & 66.98 & 122.64 & 4.5204 & 2038.000 & .000 \\
\hline Tranquilidad & 119.14 & 82.70 & 5.5714 & 2963.500 & .000 \\
\hline Satisfacción & 112.94 & 87.44 & 7.6327 & 3490.000 & .001 \\
\hline Felicidad & 129.98 & 74.40 & 7.2449 & 2042.000 & .000 \\
\hline Amor & 133.06 & 71.47 & 5.6872 & 1717.000 & .000 \\
\hline
\end{tabular}

En cuanto a los diferentes niveles de su puesta en valor. Se confirma que para cada segmento existen diferencias en relación con: ¿cacao como principal producto en los paisajes manabitas? $(p<.000)$; ¿declaratoria cultural de protección en relación con el cacao para Manabí? $(p<.018)$; y si ¿estaría dispuesto a formar parte de un circuito y/o producto turístico relacionado al paisaje cultural del cacao? $(p<.000)$. Después de realizar un Chi-cuadrado para examinar los datos nominales y una tabulación cruzada, se encuentra que esta diferencia se debe básicamente a las respuestas más altas de los productores: Cacao-Cultura con un significativo 96.5\% $(n=82)$ de apoyo a la creación de un circuito y/o producto turístico. No se evidencia diferencia en ¿mayor presencia los productos relacionados al cacao? $(p<.442)$. Aceptación parcial de la H6.

\section{Comparativa con el Paisaje Cultural del Café en Colombia}

El Paisaje Cultura del Café en Colombia fue inscrito en la UNESCO el año 2011, es el referente más cercano para Ecuador. Se le otorgó la declaratoria por cumplir exitosamente con dos de los diez criterios exigidos relacionados a generar valor agregado alrededor del patrimonio agrario de un territorio:

[primer criterio] Ser un ejemplo sobresaliente de un asentamiento humano tradicional, uso de la tierra o uso del mar que es representativo de una cultura (o culturas), o interacción humana con el medio ambiente, especialmente cuando se ha vuelto vulnerable bajo el impacto de un cambio irreversible ...

[segundo criterio] Estar asociado directa o tangiblemente con acontecimientos o tradiciones vivas, con ideas o con creencias, con obras artísticas y literarias de destacada importancia universal. (UNESCO, 2021)

Entre las características más importantes resaltan los 6 paisajes cafetaleros y 18 centros urbanos que lo conforman, con la adaptación de cultivos complejos debido a la ubicación geográfica en pequeñas parcelas cafetaleras, que lo diferencian de la producción local y lo transforman en un modelo a seguir para otras 
realidades. A esto, se suma una arquitectura tradicional adoptada en los tiempos coloniales y protegida con posterioridad. Se encuentran similitudes, pero también grandes diferencias con el territorio centro norte de la provincia de Manabí y su cultura cacaotera, ya que por un lado existe una gran diversidad en las características organolépticas del cacao originario de esta zona por la forma de cultivos asociados existentes, perfiles aromáticos y de sabores totalmente diferentes a otras zonas geográficas dentro del mismo Ecuador. Lamentablemente el tema arquitectónico tradicional es una de las grandes diferencias, ya que no existe una normativa que proteja edificaciones tradicionales o espacios productivos tradicionales.

Los valores culturales de la declaratoria de Paisaje Cultural del Café se basan, entre otras cosas, en las opciones laborales para la familia, ya que la experiencia aprendida genera un conocimiento que pasa de generación en generación, el mismo que se convierte en patrimonio. La cultura alrededor del café se transforma en una especie de capital social, que ha sido fortalecida coyunturalmente por las instituciones del estado, instituciones locales, actores privados y comunidad en general, que deben trabajar en una correcta sintonía que promueva la relación entre tradición y tecnología para garantizar la calidad y sostenibilidad del producto (Ministerio de Cultura de Colombia, 2011). Estos puntos se traducen en varias mejoras alrededor del sistema de productividad del cacao en la zona centro de Manabí, que incluye los procesos de conservación de las tradiciones y formas de cultivo únicas del entorno, que están desapareciendo resultado de la migración producto del desempleo que los afecta como ocurre en otros contextos latinoamericanos. La transferencia de conocimientos ancestrales debe ir de la mano con los avances tecnológicos, no solo para potenciar los cultivos, sino para entender los procesos culturales y la actividad turística. Es preciso contar con una red social y empresarial que sean soporte de un nuevo modelo de gestión que apunte al mejoramiento de la calidad de los productos y servicios privados, haciendo un esfuerzo considerable para la promoción turística desde lo público.

\section{Conclusiones}

Teniendo como objetivo identificar los diferentes grupos de productores, a partir de sus motivaciones, para el cultivo y producción del cacao en la zona centro norte de Manabí (Ecuador), esta investigación contribuye brindando mayor información relacionada con las motivaciones por el cultivo del cacao y con la creación de productos y/o rutas turísticas que estimulen el marketing turístico y fomenten el desarrollo territorial del turismo.

Para ello, se crea una cartografía base con información relevante de la zona, mediante la realización de capas temáticas y la georreferenciación de las fincas de cacao. Además, se realiza el mapa temático con las áreas de cultivo de cacao y los cultivos asociados de cada uno de los cantones seleccionados. La identificación de los productores encuestados permite tener una ilustración completa y ser referencia para próximos trabajos de investigación en esta región ecuatoriana.

Se establece bajo nivel de inclusión de los actores locales dentro de las propuestas de desarrollo llevadas a cabo en el territorio. Los productores no conocen acerca de proyectos ejecutados que contemplen el desarrollo de actividades culturales en las fincas o mercados cercanos y tienen escasa noción sobre la actividad turística; no obstante, muestran interés por nuevas actividades que permitan generar ingresos para sus familias. 
Se corrobora, además, que existen diferentes motivaciones para cultivar y producir el cacao en la zona centro norte de Manabí. En los resultados se evidencian dos clústeres de productores, Clúster 1: Cacao-Cultura, que representa el $43.4 \%$ de los productores y sus motivaciones más representativas son cultura, identidad y pasión; y Clúster 2: Cacao-Trabajo, que representa el 56.6\% de los productores, quienes refieren como motivaciones principales el trabajo, el negocio y el sustento diario. Las pruebas de estadística no paramétrica validaron que la segmentación de los productores explica los diferentes niveles de importancia del cacao, su cultura, utilización, consumo, percepción del cultivo y su puesta en valor. Por lo tanto, y a pesar de la carencia de una vertebración institucional, se vislumbra un contexto positivo para las iniciativas en torno al turismo agrario.

\section{Agradecimientos}

Por el financiamiento institucional de la Escuela Superior Politécnica Agropecuaria de Manabí «Manuel Félix López», mediante su proyecto institucional con registro de SENPLADES CUP; 383022, denominado: Línea base para la generación de «paisajes culturales» como nueva alternativa de desarrollo turístico de Manabí: caso de estudio el cacao. El mismo que tiene como objetivo general lo siguiente: Convertir el Patrimonio Cultural en torno al Cacao en un elemento de desarrollo económico a nivel regional y nacional; capaz de integrarse e interactuar con otras propuestas de desarrollo del sector turístico a nivel provincial en la zona centro norte de Manabí.

\section{Conflicto de intereses}

Los autores no tienen conflicto de intereses con el contenido de este trabajo.

\section{Contribución de autoría}

AGFM: Elaboración del manuscrito.

NGR: Análisis estadístico.

AFCS: Análisis de resultados y elaboración de cartografía.

WPCG: Recolección de datos.

\section{Referencias}

Andreu, L., Kozak, M., Avci, N. y Cifter, N. (2005). Market Segmentation by Motivations to Travel: British Tourists Visiting Turkey. Journal of Travel \& Tourism Marketing, 19(1), 1-14. https://doi.org/10.1300/J073v19n01

Aranda, F., Delgado, P. y Urbistondo, P. (2002). Imagen turística de las Comunidades Autónomas españolas ante una demanda segmentada. Estudios de Economía Aplicada, 20(3), 627-649.

Asociación Nacional de Exportadores e Industriales de Cacao del Ecuador - ANECACAO. (2016). Exportación ecuatoriana de cacao 2015. Recuperado el 10 de noviembre de 2019. http://www.anecacao.com/index.php/es/estadisticas/estadisticas-actuales.html. 
Barrera, V., Alwang, J., Casanova, T., Domínguez, J., Escudero, L., Loor, G., Peña, G., Párraga, J., Arévalo, J., Quiroz, J., Tarqui, O., Plaza, L., Sotomayor, I., Zambrano, F., Rodríguez, G., García, C. y Racines, M. (2019). La cadena de valor del cacao y el bienestar de los productores en la provincia de Manabí-Ecuador. Instituto Nacional de Investigaciones Agropecuarias - INIAP (Libro técnico n. ${ }^{\circ}$ 171). https://repositorio.iniap.gob.ec/handle/41000/5377

Barrezueta-Unda, S. y Paz, A. (2017). Caracterización de la sostenibilidad en función de aspectos socioeconómica del sistema agrario cacao en la provincia de El Oro, Ecuador. Revista científica Agroecosistemas, 5(1), 6-16. https://aes.ucf.edu.cu/index.php/aes

Bonilla, J. M., Boerasu, A. y Bonilla, L. M. (2014). Perfiles de los visitantes en el turismo de naturaleza: análisis exploratorio en el entorno de Doñana. International Journal of World of Tourism, 1(1), 17-25.

Carballo, R., Fraiz, J. A., Araíjo, N. y Rivo, E. (2016). Segmentación del mercado de un destino turístico de interior. El caso de A Ribeira Sacra (Ourense). PASOS. Revista de Turismo y Patrimonio Cultural, 14(2), 369-383. https://doi.org/https://doi.org/10.25145/ J.PASOS.2016.14.024

Castro, M., Barcia, M., Labrada, M. y Chasing, E. (2018). El guadúa que se corta: paisajes culturales y patrimonio construido en la costa ecuatoriana (Manabí, Ecuador). Revista Española de Antropología Americana, 48, 79-103. https://doi.org/10.5209/ REAA.63691

Cristóbal-Fransi, E., Daries-Ramon, N. y Baldomar, J. P. (2014). Segmentación de los e -consumidores: un estudio aplicado a partir de las perspectivas de uso de Internet. Cuadernos de Gestión, 14(1), 35-55. https://doi.org/10.5295/cdg.120373ec.

Duis, U. (2007). La valorización cultural, social y turística de los recursos culturales y naturales como instrumento para la planificación turística, la conservación del Paisaje Cultural Cafetero y el desarrollo sostenible del territorio turístico. Turismo y sociedad, 8, 6979.

Félix, A. (2018). Turismo y cacao: alternativa de desarrollo para las comunidades campesinas. En A. Nieto et al. Planificación turística en territorios campesinos (pp. 59-91). Corporación Universitaria UNITEC \& Escuela Superior Politécnica Agropecuaria de Manabí.

Félix, A. y Bayas, J. (2016). El agroturismo como dinamizador de destinos turísticos en el centro de Manabí. En A. Mendoza (Ed.), CONGRETUR 2016 Investigaciones turísticas del Ecuador: Fortaleciendo la innovación en los destinos turísticos (pp. 105-116). Universidad Estatal de la Península de Santa Elena.

Félix, A. y Doumet, Y. (2016). Teoría y práctica del turismo rural: casos de planificación. Abya Yala.

Félix, A., Castro, M. y Labrada, M. (2017). Investigación para el diseño de propuestas de paisajes culturales al servicio del desarrollo local. Un estudio de caso. Cuadernos de RSO, 5(2), 63-73.

Félix, A., Zepeda, A. y Villafuerte, J. (2021). Turismo en tiempo de pandemias. Covid-19 en Latinoamérica. Turismo y Sociedad, 24, 129-155. doi: https://doi.org/10.18601/01207555.n29.06

Galletto, L. (2018). A comparison between tourists’ profiles in two Italian wine routes. Revista de La Facultad de Ciencias Agrarias, 50(1), 157-170. http://revista.fca.uncu.edu.ar/images/stories/pdfs/2018-01/Cp_11_Galletto.pdf

García, N. (2021). Segmentación por motivaciones y valoración del turismo interior en el destino Manta, Manabí, Ecuador. Innovar, 31(80), 57-72. http://www.fce.unal.edu.co/media/files/innovar/Vol_31_Num_80_VF/4_Segmentacion_motivaciones_abril_16.pdf

García, M., Amores, L. E., Barquín, R. del C. y Soto, I. Y. (2017). Perfil del visitante de naturaleza en Latinoamérica: prácticas, motivaciones e imaginarios. Estudio comparativo entre México y Ecuador. Pasos Revista de Turismo y Patrimonio Cultural, 15(3), 713-729. https://doi.org/10.25145/j.pasos.2017.15.047

González, E. A. y Conde, E. M. (2011). Procedimiento para medir la demanda turística en un destino. Revista de Investigación En Turismo y Desarrollo Local TURyDES, 4(11), 1-25. http://www.eumed.net/rev/turydes/11/gacp.pdf

Gómez, A. (2015). Modelo de diseño para la valoración y apropiación social del Patrimonio en el Paisaje Cultural Cafetero colombiano. Kepes, 11(12), 118-138.

Instituto Nacional de Meteorología e Hidrología - INAMHI. (2012). Anuario Meteorológico 2010 (n. 50). Dirección de Gestión Meteorológica. https://www.inamhi.gob.ec/docum_institucion/anuarios/meteorologicos/Am\%202010.pdf 
Jiménez, J. L., García, M. M., García, M. F. y Crisol, J. A. (2010). Sistema de Información Geográfica para la generación de mapas temáticos (SIGMAT). Producción de cartografía temática. En J. Ojeda, M. F. Pita e I. Vallejo (Eds.), Tecnologías de la Información Geográfica: La Información Geográfica al servicio de los ciudadanos (pp. 1283-1294). Secretariado de Publicaciones de la Universidad de Sevilla. https://idus.us.es/bitstream/handle/11441/66781/JIMENEZ.PDF;jsessionid=FCE1E64B61 140F2D81A277709C7B614E?sequence=1

Lehmann, S. y Springer-Heinze, A. (2014). The value chain approach to smallholder development in Ecuador adopted by the German Agency for International Cooperation (GIZ). En R. Hernández, J. Martínezz-Piva y N. Mulder (Eds.), Global value chains and development (Vol. 21). ECLAC.

Lopes, S. D. (2011). Geo-segmentación y geo-posicionamiento en el análisis de las preferencias de los turistas: La geometría al servicio del marketing. Estudios y Perspectivas en Turismo, 20, 842-854. http://www.scielo.org.ar/scielo.php?script=sci_arttext\&pid=S185117322011000400005\&lang=pt\%0Ahttp://www.scielo.org.ar/pdf/eypt/v20n4/v20n4a05.pdf

MAG. (2019). Áreas de cultivo de cacao en Manabí. http://geoportal.agricultura.gob.ec/

Mayorga, D. (2015). Paisaje Cultural Cafetero, Patrimonio de la Humanidad. La cuestión del discurso patrimonial en contraste con el paisaje de la caficultura. Territorios, 32, 35-59.

Ministerio de Cultura de Colombia. (2011). Paisaje cultural cafetero: Un paisaje cultural productivo en permanente desarrollo. Nomos Impresores.

Ministerio de Turismo del Ecuador - MINTUR. (2019). Plan Nacional de Turismo. In Plan Nacional de turismo 2030. https:// www.turismo.gob.ec/wp-content/uploads/2020/03/PLAN-NACIONAL-DE-TURISMO-2030-v.-final-Registro-Oficial-sumilladocomprimido_compressed.pdf

Paguay, J. (2012). De la «Pepa de Oro» a la Ruta del Cacao. Res Non Verba, 2(2), 37-52. https://biblio.ecotec.edu.ec/revista/edicion2/ DE\%20LA\%20\%E2\%80\%9CPEPA\%20DE\%20ORO\%E2\%80\%9D\%20A\%20LA\%20RUTA\%20DEL\%20CACAO.pdf

Pardo, C. E. y Del Campo, P. (2007). Combinación de métodos factoriales y de análisis de conglomerados en R: el paquete FactoClass. Revista Colombiana de Estadística, 30(2), 231-245.

PRO ECUADOR. (2013). Análisis del sector cacao y elaborados (Dirección de Inteligencia Comercial e Inversiones). http:// infocafes.com/portal/wp-content/uploads/2016/06/PROEC_AS2013_CACAO.pdf

Quingaísa, E. y Riveros, H. (2007). Estudio de caso: Denominación de origen «Cacao Arriba». IICA Ecuador Quito Ecuador.

Quintana, M. y Aguilar, J. (2018). Denominación de origen de cacao ecuatoriano: ¿un aporte de marketing global? Innova research journal, 3(10.1), 68-76. https://revistas.uide.edu.ec/index.php/innova/article/view/825/1201

Ramirez-Anormaliza, R., Guevara-Viejo, F., Regnault, M., Pena-Holguin, R., Farias-Lema, R., Bravo-Duarte, F. y Castelo-Gónzalez, J. (2017). Análisis Multivariante: Teoría y práctica de las principales técnicas. Milagro, Ecuador: Holguín S.A. - Universidad Estatal de Milagro - UNEMI.

Rodríguez, D. y Fusco, M. (2017). Gestión de riesgos agropecuarios en el sector del cacao en Ecuador. Revista de Investigación en Modelos Financieros, 6(I), 57-74.

Teneda, W. (2016). Mejoramiento del Proceso de Fermentación del Cacao (Theobroma cacao L.) Variedad Nacional y Variedad CCN51. Universidad Internacional de Andalucía.

UNESCO. (2017). Cátedra UNESCO. Paisajes Culturales. http://www.catedraunesco.eu/

UNESCO. (2021, 28 de mayo). www.unesco.org.UNESCO. http://whc.unesco.org/en/criteria/

Vassallo, M. (2017). Diferenciación y agregado de valor en la cadena ecuatoriana del cacao. Revista Repique, 1(1), 23-43. http:// revistasdigitales.utelvt.edu.ec/revista/index.php/repique/article/view/2

Vela, A. (2016). «El Cacao: Identidad, Cultura y Gastronomía Ecuatoriana» Ensayo acerca de un análisis retrospectivo, actual y prospectivo del cacao en Ecuador. [Tesis de grado, Universidad de los Hemisferios]. Quito.

Zambrano, C. (2014). La segunda Revolución Industrial europea, el valle de Chone y el cacao. Revista Cultural Spondylus, 40, 5-25. 
Ángel Guillermo Félix Mendoza

Coordinador del grupo de investigación GISTUR de la Escuela Superior Politécnica Agropecuaria de Manabí "Manuel Félix López" (ESPAM MFL), Ecuador. Ph. D. (c) Programa de recursos naturales y gestión sostenible, Universidad de Córdoba (UCO). Magister en planificación y gestión de proyectos agroturísticos y ecológicos, Universidad Agraria del Ecuador (UAE). Ingeniero en administración de empresas turísticas y hoteleras, Universidad Laica Eloy Alfaro de Manabí (ULEAM). Docente investigador desde el 2015. Investigador acreditado por Secretaría de Educación Superior, Ciencia, Tecnología e Innovación (SENESCYT). Director de proyectos de colaboración interinstitucional, editor de la revista RIGISTUR.

guillofelix@gmail.com, afelix@espam.edu.ec

ORCID https://orcid.org/0000-0003-1586-1068

Nelson García Reinoso

Coordinador de la maestría se turismo de la Escuela Superior Politécnica Agropecuaria de Manabí "Manuel Félix López" (ESPAM MFL), Ecuador.

Ph. D. (c) Doctorado interuniversitario en turismo, Universidad de Alicante (EDUA). Máster en gestión turística, Universidad Central de las Villas (UCVL). Docente investigador y director de proyectos de investigación del Grupo GISTUR, desde el 2016. Investigador acreditado por Secretaría de Educación Superior, Ciencia, Tecnología e Innovación (SENESCYT). Coordinador académico de la carrera de licenciatura en turismo en ESPAM MFL.

ngarcia@espam.edu.ec

ORCID: https://orcid.org/0000-0003-0972-3347

Ángel Frowen Cedeño Sacón

Docente de la carrera de Ingeniería Agrícola de la Escuela Superior Politécnica Agropecuaria de Manabí "Manuel Félix López" (ESPAM MFL), Ecuador. Ph. D. (c) Programa de recursos naturales y gestión sostenible,Universidad de Córdoba (UCO). Master en Ciencias de la geo información y observación de la tierra. Mención información de la tierra para la planificación del territorio, Universidad Mayor de San Simón (UMSS). Ingeniero Agrícola, Universidad Técnica de Manabí (UTM), desde el 2016. Investigador acreditado por Secretaría de Educación Superior, Ciencia, Tecnología e Innovación (SENESCYT). Director de proyectos en la ESPAM MFL.

acedeno@espam.edu.ec

ORCID: https://orcid.org/0000-0002-7589-3611

\section{Wilson Paúl Cedeño Guzmán}

Director de la unidad de docencia, investigación y vinculación de cacao Escuela Superior Politécnica Agropecuaria de Manabí "Manuel Félix López" (ESPAM MFL), Ecuador.

Magister en agroindustria, Escuela Superior Politécnica Agropecuaria de Manabí Manuel Félix López. Ingeniero agroindustrial, ESPAM MFL. Tecnólogo en agroindustrias, ESPAM MFL. Docente auxiliar e investigador de la carrera de agroindustrias ESPAM MFL. Especialista en la catación de chocolate formado por especialistas del Salón del Chocolate de Europa.

wpcedeno@espam.edu.ec

ORCID: https://orcid.org/0000-0002-9506-9777 